\title{
A note on $G$-preinvex functions
}

\author{
Xiaoling Liu1, Dehui Yuan ${ }^{1 *}$ and Chuanqing $\mathrm{Xu}^{2}$
}

${ }^{\text {"Correspondence: }}$

dhyuan@hstc.edu.cn

${ }^{1}$ Department of Mathematics,

Hanshan Normal University,

Chaozhou, Guangdong 521041,

China

Full list of author information is

available at the end of the article

\begin{abstract}
With the equivalent relationships between the G-generalized invexities and general invexities on the hand, we present two characterizations for G-preinvexity; we also discuss the relationships between different $G$-generalized invexities such as G-preinvexity, strict G-preinvexity and semistrict G-preinvexity. Note that our results are proved by applying the results from general invexities introduced in the literatures.
\end{abstract}

Keywords: invex set; G-generalized invexity; invexity

\section{Introduction}

Recently, Antczak [1, 2] introduced the concept of the G-preinvexity, which included the preinvexity [3] and the $r$-preinvexity [4] as special cases. Relation of this $G$-preinvexity to preinvexity and some properties of this class of functions were studied in [2]. In another recent paper, Luo and Wu [5] introduced a new class of functions, named semistrictly $G$-preinvex functions. The relationships between semistrictly G-preinvex functions and $G$-preinvex functions were investigated under mild assumptions. Their results improved and extended the existing ones in the literature. Also, the properties of semistrictly $G$-preinvex functions were further considered by Peng in [6].

In this note, we are interested in the relationships between three kinds of $G$-generalized invexities. For this purpose, we firstly investigate the relation between the $G$-generalized invexities and the corresponding general generalized invexities. Then we characterize these $G$-generalized invexities by applying the well-known results from the preinvexity, the strict preinvexity and the semistrict preinvexity. Moreover, we point out that our method is different from the one used by Luo and $\mathrm{Wu}$ in [5]. The rest of this note is organized as follows. In Section 2, we give some definitions and some preliminaries; moreover, we establish the useful Lemma 1. Section 3 presents two characterizations for G-preinvex functions and proves that, under certain conditions, the $G$-preinvexity is equivalent with prequasi-invexity when an intermediate-point G-preinvexity is required. In Section 4, we obtain relationships between different $G$-generalized invexities. Section 5 gives some conclusions.

\section{Definitions and preliminaries}

In this section, we provide some definitions and some notations. Moreover, we establish an important lemma.

() 2013 Liu et al.; licensee Springer. This is an Open Access article distributed under the terms of the Creative Commons Attribution License (http://creativecommons.org/licenses/by/2.0), which permits unrestricted use, distribution, and reproduction in any medium, provided the original work is properly cited. 
Definition 1 [3] Let $X \subset \mathbb{R}^{n}, \eta: \mathbb{R}^{n} \times \mathbb{R}^{n} \rightarrow \mathbb{R}^{n}$. The set $X$ is said to be invex at $u \in X$ with respect to $\eta$ if for all $x \in X$ such that

$$
u+\lambda \eta(x, u) \in X, \quad \forall \lambda \in[0,1] .
$$

$X$ is said to be invex set with respect to $\eta$ if $X$ is invex at each $u \in X$.

Definition 2 [7] Let $X$ be a nonempty invex subset of $\mathbb{R}^{n}$ with respect to $\eta$. A function $f: X \rightarrow \mathbb{R}$ is said to be preinvex at $u \in X$ with respect to $\eta$ if

$$
f(u+\lambda \eta(x, u)) \leq \lambda f(x)+(1-\lambda) f(u), \quad \forall \lambda \in[0,1], \forall x \in X .
$$

The function $f$ is said to be preinvex on $X$ with respect to $\eta$ if $f$ is preinvex at each $u \in X$ with respect to $\eta ; f$ is said to be strictly preinvex on $X$ with respect to $\eta$ if the inequality (1) strictly holds for all $x, u \in X$ such that $x \neq u ; f$ is said to be semistrictly preinvex on $X$ with respect to $\eta$ if the inequality (1) strictly holds for all $x, u \in X$ such that $f(x) \neq f(u)$.

Definition $3[1,2,5]$ Let $X$ be a nonempty invex subset of $\mathbb{R}^{n}$ with respect to $\eta$. A function $f: X \rightarrow \mathbb{R}$ is said to be $G$-preinvex at $u$ on $X$ with respect to $\eta$ if there exists a continuous function $G: \mathbb{R} \rightarrow \mathbb{R}$ such that $G: I_{f}(X) \rightarrow \mathbb{R}$ is a strictly increasing function on its domain, and

$$
f(u+\lambda \eta(x, u)) \leq G^{-1}(\lambda G(f(x))+(1-\lambda) G(f(u))), \quad \forall \lambda \in[0,1], \forall x \in X .
$$

The function $f$ is said to be $G$-preinvex on $X$ with respect to $\eta$ if $f$ is $G$-preinvex at each $u \in$ $X$ with respect to $\eta ; f$ is said to be strictly $G$-preinvex on $X$ with respect to $\eta$ if the inequality (2) strictly holds for all $x, u \in X$ such that $x \neq u ; f$ is said to be semistrictly $G$-preinvex on $X$ with respect to $\eta$ if the inequality (2) strictly holds for all $x, u \in X$ such that $f(x) \neq f(u)$.

From Definition 3, $G$ is a strictly increasing function because $G^{-1}$ must exist. Hence, let $G$ be a strictly increasing function throughout this note. Now we present a useful lemma.

Lemma 1 Let $f: X \rightarrow \mathbb{R}$. Suppose $G: I_{f}(X) \rightarrow \mathbb{R}$ is a strictly increasing function on its domain. Then

(i) $f$ is G-preinvex on $X$ with respect to $\eta$ if and only if $G(f)$ is preinvex on $X$ with respect to $\eta$;

(ii) $f$ is strictly G-preinvex on $X$ with respect to $\eta$ if and only if $G(f)$ is strictly preinvex on $X$ with respect to $\eta$;

(iii) $f$ is semistrictly G-preinvex on $X$ with respect to $\eta$ if and only if $G(f)$ is semistrictly preinvex on $X$ with respect to $\eta$.

Proof (i) By the monotonicity of $G$, we know that the inequality (2) is equivalent to

$$
G(f(u+\lambda \eta(x, u))) \leq \lambda G(f(x))+(1-\lambda) G(f(u)), \quad \forall \lambda \in[0,1], \forall x \in X .
$$

Therefore, by Definitions 2 and 3, $f$ is $G$-preinvex on $X$ with respect to $\eta$ if and only if $G(f)$ is preinvex on $X$ with respect to $\eta$.

Similar to part (i), we can prove part (ii) and (iii). 


\section{Semicontinuity and G-preinvexity}

In this section, two conditions that determine the $G$-preinvexity of a function via an intermediate-point G-preinvexity check under conditions of upper and lower semicontinuity, respectively, are presented; moreover, equivalent relationship between $G$ preinvexity and prequasi-invexity is proved under the intermediate-point $G$-preinvexity assumption. Here, we need the following Condition $C$, which was introduced by Mohan and Neogy in [8]. The function $\eta: \mathbb{R}^{n} \times \mathbb{R}^{n} \rightarrow \mathbb{R}^{n}$ satisfies Condition $C$ if

$$
\begin{aligned}
& \eta(y, y+\lambda \eta(x, y))=-\lambda \eta(x, y), \\
& \eta(x, y+\lambda \eta(x, y))=(1-\lambda) \eta(x, y)
\end{aligned}
$$

hold for any $x, y \in X$ and for any $\lambda \in[0,1]$.

The upper and lower semicontinuity of a real function $f$ is defined as follows.

Definition 4 [9] Let $X$ be a nonempty subset of $\mathbb{R}^{n}$. A function $f: X \rightarrow \mathbb{R}$ is said to be upper semicontinuous at $\bar{x} \in X$ if, for every $\epsilon>0$, there exists a $\delta>0$ such that for all $x \in X$, if $\|x-\bar{x}\|<\delta$, then

$$
f(x)<f(\bar{x})+\epsilon \text {. }
$$

If $-f$ is upper semicontinuous at $\bar{x} \in X$, then $f$ is said to be lower semicontinuous at $\bar{x} \in X$.

We also need the following Lemma 2, which is Lemma 3.2 in [9].

Lemma 2 Let $X$ be a nonempty, open and invex set in $\mathbb{R}^{n}$ with respect to $\eta: \mathbb{R}^{n} \times \mathbb{R}^{n} \rightarrow \mathbb{R}^{n}$, where $\eta$ satisfies Condition C. Assume that $f: X \rightarrow \mathbb{R}$ satisfies

$$
f(y+\eta(x, y)) \leq f(x), \quad \forall x, y \in X
$$

Moreover, there exists an $\alpha \in(0,1)$ such that for every $x, y \in X$ the inequality

$$
f(y+\alpha \eta(x, y)) \leq \alpha f(x)+(1-\alpha) f(y)
$$

holds. Then the set $A:=\{\lambda \in[0,1] \mid f(y+\lambda \eta(x, y)) \leq \lambda f(x)+(1-\lambda) f(y), \forall x, y \in X\}$ is dense in $[0,1]$.

Under semicontinuity conditions, Yang proved from Lemma 2 that judging a function to be preinvex or not can be reduced to checking intermediate-point preinvexity for the function; see the following Lemmas 3 and 4, which are taken from Theorems 3.1 and 3.2 in [9], respectively.

Lemma 3 Let $X$ be a nonempty open invex set in $\mathbb{R}^{n}$ with respect to $\eta: \mathbb{R}^{n} \times \mathbb{R}^{n} \rightarrow \mathbb{R}^{n}$, where $\eta$ satisfies Condition C. Assume that the function $f: X \rightarrow \mathbb{R}$ is upper semicontinuous on $X$ and satisfies

$$
f(y+\eta(x, y)) \leq f(x), \quad \forall x, y \in X .
$$


Then $f$ is a preinvex function on $X$ if and only if there exists an $\alpha \in(0,1)$ such that

$$
f(y+\alpha \eta(x, y)) \leq \alpha f(x)+(1-\alpha) f(y), \quad \forall x, y \in X .
$$

Lemma 4 Let $X$ be a nonempty invex set in $\mathbb{R}^{n}$ with respect to $\eta: \mathbb{R}^{n} \times \mathbb{R}^{n} \rightarrow \mathbb{R}^{n}$, where $\eta$ satisfies Condition C. Assume that the function $f: X \rightarrow \mathbb{R}$ is lower semicontinuous on $X$ and satisfies

$$
f(y+\eta(x, y)) \leq f(x), \quad \forall x, y \in X
$$

Then $f$ is a preinvex function on $X$ if and only iffor any $x, y \in X$, there exists an $\alpha \in(0,1)$ such that

$$
f(y+\alpha \eta(x, y)) \leq \alpha f(x)+(1-\alpha) f(y) .
$$

With Lemmas 2-4 on hand, we can prove the following Theorems 1-3, respectively.

Theorem 1 Let $X$ be a nonempty invex set in $\mathbb{R}^{n}$ with respect to $\eta: \mathbb{R}^{n} \times \mathbb{R}^{n} \rightarrow \mathbb{R}^{n}$, where $\eta$ satisfies Condition C. Assume that $f: X \rightarrow \mathbb{R}$ satisfies

$$
f(y+\eta(x, y)) \leq f(x), \quad \forall x, y \in X
$$

Suppose the function $G$ is increasing on $I_{f}(X)$. Moreover, there exists an $\alpha \in(0,1)$ such that for every $x, y \in X$ the inequality

$$
G(f(y+\alpha \eta(x, y))) \leq \alpha G(f(x))+(1-\alpha) G(f(y))
$$

holds. Then the set $A:=\{\lambda \in[0,1] \mid G(f(y+\lambda \eta(x, y))) \leq \lambda G(f(x))+(1-\lambda) G(f(y)), \forall x, y \in X\}$ is dense in $[0,1]$.

Proof From the assumption of this theorem, we have

$$
G(f(y+\eta(x, y))) \leq G(f(x)), \quad \forall x, y \in X .
$$

Hence, we can deduce the result from Lemma 2.

Theorem 2 Let $X$ be a nonempty open invex set in $\mathbb{R}^{n}$ with respect to $\eta: \mathbb{R}^{n} \times \mathbb{R}^{n} \rightarrow \mathbb{R}^{n}$, where $\eta$ satisfies Condition C. Assume that a function $f: X \rightarrow \mathbb{R}$ is upper semicontinuous on $X$ and satisfies

$$
f(y+\eta(x, y)) \leq f(x), \quad \forall x, y \in X .
$$

Moreover, the function $G$ is both continuous and increasing on $I_{f}(X)$. Then $f$ is G-preinvex on $X$ if and only if there exists an $\alpha \in(0,1)$ such that

$$
G(f(y+\alpha \eta(x, y))) \leq \alpha G(f(x))+(1-\alpha) G(f(y)), \quad \forall x, y \in X .
$$


Proof By assumption, we know that the function $G(f)$ is upper semicontinuous on $X$ and it satisfies

$$
G(f(y+\eta(x, y))) \leq G(f(x)), \quad \forall x, y \in X .
$$

Replacing $f$ by $G(f)$ in Lemma 3 and combining Lemma 1(i), we obtain the desired result.

If $f$ is continuous on $X$, then the above Theorem 2 is Theorem 10 in [1]. However, our proof is simpler than the proof of Theorem 10 in [1], since we apply the result pertaining to the preinvexity as defined in Definition 2.

Theorem 3 Let $X$ be a nonempty invex set in $\mathbb{R}^{n}$ with respect to $\eta: \mathbb{R}^{n} \times \mathbb{R}^{n} \rightarrow \mathbb{R}^{n}$, where $\eta$ satisfies Condition $C$. Assume that the function $f: X \rightarrow \mathbb{R}$ is lower semicontinuous on $X$ and satisfies

$$
f(y+\eta(x, y)) \leq f(x), \quad \forall x, y \in X .
$$

Moreover, the function $G$ is both continuous and increasing on $I_{f}(X)$. Then $f$ is $G$-preinvex on $X$ if and only iffor any $x, y \in X$, there exists an $\alpha \in(0,1)$ such that

$$
G(f(y+\alpha \eta(x, y))) \leq \alpha G(f(x))+(1-\alpha) G(f(y)) .
$$

Proof By the assumption of the theorem, it is easy to check that

$$
G(f(y+\eta(x, y))) \leq G(f(x)), \quad \forall x, y \in X .
$$

Moreover, $G(f)$ is lower semicontinuous on $X$. Now, with Lemma 1(i) and Lemma 4, we derive the desired result.

The above Theorems 2 and 3 illustrate that, to justify G-preinvexity of a function, it is sufficient to check intermediate-point $G$-preinvexity for the function. Our development extends the results of general preinvexity to the G-preinvexity. Note that Theorems 1-3 generalize Lemmas 2-4 from the preinvex case to the G-preinvex situation, respectively.

On the relationship between the preinvexity and prequasi-invexity, where the prequasiinvexity concept is presented in Definition 5, Yang et al. obtained an interesting result (see Lemma 5).

Definition 5 Let $X$ be a nonempty invex subset of $\mathbb{R}^{n}$ with respect to $\eta$. A function $f$ : $X \rightarrow \mathbb{R}$ is said to be prequasi-invex on $X$ if

$$
f(y+\lambda \eta(x, y)) \leq \max \{f(x), f(y)\}, \quad \forall \lambda \in[0,1], \forall x, y \in X .
$$

Remark 1 If the function $G$ is strictly increasing on $I_{f}(X)$, then $f$ is prequasi-invex on $X$ if and only if $G_{f}(f)$ is prequasi-invex on $X$.

Lemma 5 [9] Let $X$ be a nonempty invex set in $\mathbb{R}^{n}$ with respect to $\eta: \mathbb{R}^{n} \times \mathbb{R}^{n} \rightarrow \mathbb{R}^{n}$, where $\eta$ satisfies Condition $C$. Then a function $f: X \rightarrow \mathbb{R}$ is preinvex on $X$ if and only if it is a 
prequasi-invex function on $X$ and there exists an $\alpha \in(0,1)$ such that

$$
f(y+\alpha \eta(x, y)) \leq \alpha f(x)+(1-\alpha) f(y), \quad \forall x, y \in X
$$

Next, we extend the result obtained by Yang et al. to the G-preinvex situation in the following theorem, which reveals that, under an intermediate-point G-preinvexity assumption, the $G$-preinvexity is equivalent with prequasi-invexity.

Theorem 4 Let $X$ be a nonempty invex set in $\mathbb{R}^{n}$ with respect to $\eta: \mathbb{R}^{n} \times \mathbb{R}^{n} \rightarrow \mathbb{R}^{n}$ and $f: X \rightarrow \mathbb{R}$, where $\eta$ satisfies Condition $C$. Suppose that $G$ is a strictly increasing function on $I_{f}(X)$. Then $f$ is a G-preinvex function on $X$ if and only if it is a prequasi-invex function on $X$ and there exists an $\alpha \in(0,1)$ such that

$$
G(f(y+\alpha \eta(x, y))) \leq \alpha G(f(x))+(1-\alpha) G(f(y)), \quad \forall x, y \in X
$$

Proof By Remark 1, one obtains that $f$ is a prequasi-invex function on $X$ if and only if $G(f)$ is a prequasi-invex function on $X$. Thus, we have the desired result from Lemma 1(i) and Lemma 5.

\section{Relationships among G-generalized preinvexities}

In this section, we discuss the relationships between $G$-invexities under Condition $C$. To this end, we will use the following results proved in the literatures.

Theorem 5 [10, Theorem 2.3] Let $X$ be nonempty invex set in $\mathbb{R}^{n}$ with respect to $\eta: \mathbb{R}^{n} \times$ $\mathbb{R}^{n} \rightarrow \mathbb{R}^{n}$, where $\eta$ satisfies Condition $C$. Suppose function $f: X \rightarrow \mathbb{R}$ is semistrictly preinvex on $X$ with respect to $\eta$. If there exists $a \lambda \in(0,1)$ such that

$$
f(y+\lambda \eta(x, y)) \leq \lambda f(x)+(1-\lambda) f(y), \quad \forall x, y \in X,
$$

then $f$ is a preinvex function on $X$ with respect to the same $\eta$.

Theorem 6 [10, Theorem 2.5] Let $X$ be nonempty invex set in $\mathbb{R}^{n}$ with respect to $\eta: \mathbb{R}^{n} \times$ $\mathbb{R}^{n} \rightarrow \mathbb{R}^{n}$, where $\eta$ satisfies Condition C. Suppose that $f: X \rightarrow \mathbb{R}$ is a preinvex function on $X$ with respect to $\eta$. For each pair $x, y \in X, x \neq y$, if there exists $a \lambda \in(0,1)$ such that

$$
f(y+\lambda \eta(x, y))<\lambda f(x)+(1-\lambda) f(y), \quad \forall x, y \in X,
$$

then $f$ is a strictly preinvex function on $X$ with respect to the same $\eta$.

Theorem 7 [7, Theorem 4.2] Let $X$ be a nonempty invex set in $\mathbb{R}^{n}$ with respect to $\eta: \mathbb{R}^{n} \times$ $\mathbb{R}^{n} \rightarrow \mathbb{R}^{n}$, where $\eta$ satisfies Condition $C$. Assume that $f: X \rightarrow \mathbb{R}$ is a preinvex function on $X$ with respect to $\eta$. If there exists $a \lambda \in(0,1)$ such that for every $x, y \in X, f(x) \neq f(y)$, the inequalities

$$
\begin{aligned}
& f(y+\lambda \eta(x, y))<\lambda f(x)+(1-\lambda) f(y), \\
& f(y+(1-\alpha) \eta(x, y))<\alpha f(y)+(1-\alpha) f(x)
\end{aligned}
$$

hold, then $f$ is a semistrictly preinvex function on $X$ with respect to the same $\eta$. 
Theorem 8 Let $X$ be a nonempty invex set in $\mathbb{R}^{n}$ with respect to $\eta: \mathbb{R}^{n} \times \mathbb{R}^{n} \rightarrow \mathbb{R}^{n}$, where $\eta$ satisfies Condition C. Suppose that $f: X \rightarrow \mathbb{R}$ is a semistrictly G-preinvex function on $X$ with respect to $\eta$. If there exists a $\lambda \in(0,1)$ such that

$$
G(f(y+\lambda \eta(x, y))) \leq \lambda G(f(x))+(1-\lambda) G(f(y)), \quad \forall x, y \in X,
$$

then $f$ is a G-preinvex function on $X$ with respect to the same $\eta$.

Proof Since $f$ is a semistrictly G-preinvex function on $X$ with respect to $\eta$. Then, by Lemma 1(iii), $G(f)$ is a semistrictly preinvex function on $X$ with respect to $\eta$. Replacing $f$ by $G(f)$ in Theorem 5, we deduce that $G(f)$ is a preinvex function on $X$ with respect to $\eta$. Again, from Lemma 1(i), $f$ is a $G$-preinvex function on $X$ with respect to the same $\eta$.

Recall that Theorem 8 was also presented in [5]. But our method of proof is different from [5]. Note that we establish the result by applying the above Theorem 8 , which is an existed result for semistrictly preinvex function.

Theorem 9 Let $X$ be a nonempty invex set in $\mathbb{R}^{n}$ with respect to $\eta: \mathbb{R}^{n} \times \mathbb{R}^{n} \rightarrow \mathbb{R}^{n}$, where $\eta$ satisfies Condition C. Suppose that $f: X \rightarrow \mathbb{R}$ is a G-preinvex function on $X$ with respect to $\eta$. For each pair $x, y \in X, x \neq y$, if there exists $a \lambda \in(0,1)$ such that

$$
G(f(y+\lambda \eta(x, y)))<\lambda G(f(x))+(1-\lambda) G(f(y))
$$

then $f$ is a strictly G-preinvex function on $X$ with respect to the same $\eta$.

Proof Note that $f$ is G-preinvex on $X$ with respect to $\eta$. By Lemma 1(i), $G(f)$ is preinvex on $X$ with respect to $\eta$. Now, we deduce from Theorem 6 that $G(f)$ is strictly preinvex on $X$ with respect to $\eta$. Therefore, one obtains from Lemma 1(ii) that $f$ is strictly $G$-preinvex on $X$ with respect to the same $\eta$.

Lemma 6 Let $X$ be a nonempty invex set in $\mathbb{R}^{n}$ with respect to $\eta: \mathbb{R}^{n} \times \mathbb{R}^{n} \rightarrow \mathbb{R}^{n}$, where $\eta$ satisfies Condition C. Suppose function $f: X \rightarrow \mathbb{R}$ is G-preinvex on $X$ with respect to $\eta$. If there exists an $\alpha \in(0,1)$ such that for every $x, y \in X, f(x) \neq f(y)$,

$$
G(f(y+\alpha \eta(x, y)))<\alpha G(f(x))+(1-\alpha) G(f(y)) .
$$

Then for every $x, y \in X, f(x) \neq f(y)$

$$
G(f(y+(1-\alpha) \eta(x, y)))<\alpha G(f(y))+(1-\alpha) G(f(x)) .
$$

Proof (i) If $\alpha=\frac{1}{2}$, the inequality (8) is the inequality (9).

(ii) If $\alpha<\frac{1}{2}$, then $\alpha<1-\alpha<1$. Denote by $u_{1}=1, u_{2}=\alpha$ and $\beta=\frac{1-2 \alpha}{1-\alpha}$, then $1-\alpha=$ $\beta u_{1}+(1-\beta) u_{2}$. 
From Condition C, we have

$$
\begin{aligned}
y & +u_{2} \eta(x, y)+\beta \eta\left(y+u_{1} \eta(x, y), y+u_{2} \eta(x, y)\right) \\
& =y+u_{2} \eta(x, y)+\beta \eta\left(y+u_{1} \eta(x, y), y+u_{1} \eta(x, y)-\left(u_{1}-u_{2}\right) \eta(x, y)\right) \\
& =y+u_{2} \eta(x, y)+\beta \eta\left(y+u_{1} \eta(x, y), y+u_{1} \eta(x, y)+\frac{u_{1}-u_{2}}{u_{1}} \eta\left(y, y+u_{1} \eta(x, y)\right)\right) \\
& =y+u_{2} \eta(x, y)-\beta \frac{u_{1}-u_{2}}{u_{1}} \eta\left(y, y+u_{1} \eta(x, y)\right) \\
& =y+\left(u_{2}+\beta\left(u_{1}-u_{2}\right)\right) \eta(x, y)=y+(1-\alpha) \eta(x, y) .
\end{aligned}
$$

Note that the identity (3) in Condition $C$ is used in the second, third and fourth equalities. Hence, from (8) and the $G$-preinvexity of $f$, we obtain

$$
\begin{aligned}
G( & (y+(1-\alpha) \eta(x, y))) \\
& =G\left(f\left(y+u_{2} \eta(x, y)+\beta \eta\left(y+u_{1} \eta(x, y), y+u_{2} \eta(x, y)\right)\right)\right) \\
& \leq \beta G\left(f\left(y+u_{1} \eta(x, y)\right)\right)+(1-\beta) G\left(f\left(y+u_{2} \eta(x, y)\right)\right) \\
& <\beta\left(u_{1} G(f(x))+\left(1-u_{1}\right) G(f(y))\right)+(1-\beta)\left(u_{2} G(f(x))+\left(1-u_{2}\right) G(f(y))\right) \\
& =(1-\alpha) G(f(x))+\alpha G(f(y)) .
\end{aligned}
$$

(iii) If $\alpha>\frac{1}{2}$, then $0<1-\alpha<\alpha$. Denote by $u_{1}=\alpha, u_{2}=0$ and $\beta=\frac{1-\alpha}{\alpha}$, then $1-\alpha=$ $\beta u_{1}+(1-\beta) u_{2}$. Similar to (ii), we can prove that the inequality (9) still holds.

Theorem 10 Let $X$ be a nonempty invex set in $\mathbb{R}^{n}$ with respect to $\eta: \mathbb{R}^{n} \times \mathbb{R}^{n} \rightarrow \mathbb{R}^{n}$, where $\eta$ satisfies Condition C. Assume that $f: X \rightarrow \mathbb{R}$ is a G-preinvex function on $X$ with respect to $\eta$. If there exists a $\lambda \in(0,1)$ such that for every $x, y \in X, f(x) \neq f(y)$, the inequality

$$
G(f(y+\lambda \eta(x, y)))<\lambda G(f(x))+(1-\lambda) G(f(y))
$$

holds, then $f$ is a semistrictly $G$-preinvex function on $X$ with respect to the same $\eta$.

Proof By Lemma 1(iii), it is sufficient to prove that $G(f)$ is semistrictly preinvex on $X$ with respect to $\eta$. From Lemma 6 and the assumption of Theorem 10, we know that the assumption of Theorem 7 holds. Using Theorem 7 and Lemma 1(iii), we can deduce the result.

Theorem 11 Let $X$ be a nonempty invex set in $\mathbb{R}^{n}$ with respect to $\eta: X \times X \rightarrow \mathbb{R}^{n}$, where $\eta$ satisfies Condition $\mathrm{C}$; suppose the function $f: X \rightarrow \mathbb{R}$ is lower semicontinuous and satisfies

$$
f(y+\eta(x, y)) \leq f(x)
$$

for any $x, y \in X$. Moreover, the function $G$ is both continuous and increasing on $I_{f}(X)$. If there exists an $\alpha \in(0,1)$ such that for every $x, y \in X, f(x) \neq f(y)$, the inequality

$$
G(f(y+\alpha \eta(x, y)))<\alpha G(f(x))+(1-\alpha) G(f(y))
$$

holds, then $f$ is both G-preinvex and semistrictly G-preinvex on X. 
Proof Firstly, we shall prove that $f$ is a G-preinvex function on $X$. Recalling Theorem 4, we need to show that there exists a $\lambda \in(0,1)$ such that for every $x, y \in X$ the inequality (7) holds. Assume, by a contradiction, there exist $x, y \in X$ such that

$$
G(f(y+\lambda \eta(x, y)))>\lambda G(f(x))+(1-\lambda) G(f(y)), \quad \forall \lambda \in(0,1) .
$$

Since $G$ is both continuous and increasing, then $G(f)$ is lower semicontinuous and satisfies

$$
G(f(y+\eta(x, y))) \leq G(f(x)) .
$$

We need to consider the following cases.

Case (i) $f(x) \neq f(y)$. According to (11), we must have

$$
G(f(y+\alpha \eta(x, y)))<\alpha G(f(x))+(1-\alpha) G(f(y)),
$$

which contradicts to (12).

Case (ii) $f(x)=f(y)$. Since $\alpha \in(0,1)$, then $2 \alpha-\alpha^{2}=1-(\alpha-1)^{2} \in(0,1)$. Let $\lambda=2 \alpha-\alpha^{2}$ and $\lambda=\alpha$ in (12), respectively. Then we have

$$
\begin{aligned}
& G\left(f\left(y+\left(2 \alpha-\alpha^{2}\right) \eta(x, y)\right)\right)>G(f(x)), \\
& G(f(y+\alpha \eta(x, y)))>G(f(x)) .
\end{aligned}
$$

From (14), we obtain $G(f(y+\alpha \eta(x, y))) \neq G(f(x))$. Therefore, according to (11), we obtain from Condition $\mathrm{C}$ the following inequality:

$$
\begin{aligned}
G( & \left.\left(y+\left(2 \alpha-\alpha^{2}\right) \eta(x, y)\right)\right) \\
& =G(f(y+\alpha \eta(x, y)+\alpha \eta(x, y+\alpha \eta(x, y)))) \\
& <(1-\alpha) G(f(y+\alpha \eta(x, y)))+\alpha G(f(x)) \\
& <(1-\alpha)((1-\alpha) G(f(y))+\alpha G(f(x)))+\alpha G(f(x)) \\
& =[(1-\alpha)(1-\alpha)+(1-\alpha) \alpha+\alpha] G(f(x))=G(f(x)),
\end{aligned}
$$

which contradicts to (13). Therefore, from Theorem $4, f$ is a $G$-preinvex function on $X$ with respect to $\eta$.

Further, from the above Theorem 10, $f$ is also a semistrictly G-preinvex function on $X$ with respect to $\eta$.

\section{Conclusions}

In this note, our purpose is to investigate the G-generalized invexities introduced by researchers in the past few years. To apply the existed results from the general invexities to deal with the $G$-generalized ones, we have established the useful Lemma 1, which discloses the relationships between $G$-generalized invexities and the general invexities. With this important lemma on hand, we have extended the acknowledged results pertaining to the general invexities to the corresponding G-generalized invexities. More exactly, some 
characterizations for G-preinvex functions have been deduced; when an intermediatepoint G-preinvexity is satisfied, two equivalent relationships between G-preinvexity and prequasi-invexity have been established (see Theorems 2 and 3 ). Using the existed results (Theorems 5, 6 and 7) relating to the general invexities, we deduce the similar results for G-generalized invexities; see Theorems 8, 10 and 11. Note that Theorems 8, 10 and 11 are also presented in [5]. However, our method is different from the one used by Luo and $\mathrm{Wu}$ in [5]. Here, we prove the results by applying the well-known results of the general invexities presented in the literatures.

\section{Competing interests}

The authors declare that they have no competing interests.

\section{Authors' contributions}

All authors carried out the proof. XL and DY conceived of the study, and participated in its design and coordination; CX polished the English of the manuscript. All authors read and approved the final manuscript.

\section{Author details}

'Department of Mathematics, Hanshan Normal University, Chaozhou, Guangdong 521041, China. ${ }^{2}$ Science College, Beijing University of Civil Engineering and Architecture, Beijing, 102616, China.

\section{Acknowledgements}

The authors are grateful to the referees for their valuable suggestions that helped to improve the paper in its present form. This research is supported by Science Foundation of Hanshan Normal University (LT200801).

Received: 6 October 2012 Accepted: 28 March 2013 Published: 12 April 2013

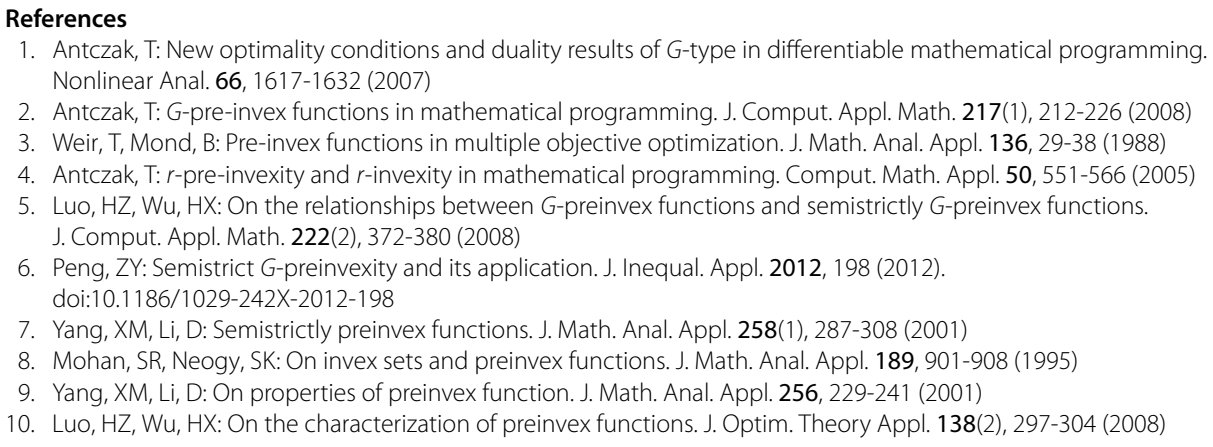

1. Antczak, T: New optimality conditions and duality results of G-type in differentiable mathematical programming. Nonlinear Anal. 66, 1617-1632 (2007)

2. Antczak, T: G-pre-invex functions in mathematical programming. J. Comput. Appl. Math. 217(1), 212-226 (2008)

3. Weir, T, Mond, B: Pre-invex functions in multiple objective optimization. J. Math. Anal. Appl. 136, $29-38$ (1988)

4. Antczak, T: r-pre-invexity and $r$-invexity in mathematical programming. Comput. Math. Appl. 50, 551-566 (2005)

5. Luo, HZ, Wu, HX: On the relationships between G-preinvex functions and semistrictly G-preinvex functions. J. Comput. Appl. Math. 222(2), 372-380 (2008)

6. Peng, ZY: Semistrict G-preinvexity and its application. J. Inequal. Appl. 2012, 198 (2012). doi:10.1186/1029-242X-2012-198

7. Yang, XM, Li, D: Semistrictly preinvex functions. J. Math. Anal. Appl. 258(1), 287-308 (2001)

8. Mohan, SR, Neogy, SK: On invex sets and preinvex functions. J. Math. Anal. Appl. 189, 901-908 (1995)

9. Yang, XM, Li, D: On properties of preinvex function. J. Math. Anal. Appl. 256, 229-241 (2001)

10. Luo, HZ, Wu, HX: On the characterization of preinvex functions. J. Optim. Theory Appl. 138(2), 297-304 (2008)

doi:10.1186/1029-242X-2013-169

Cite this article as: Liu et al.: A note on G-preinvex functions. Journal of Inequalities and Applications 2013 2013:169.

\section{Submit your manuscript to a SpringerOpen ${ }^{\circ}$ journal and benefit from:}

- Convenient online submission

Rigorous peer review

- Immediate publication on acceptance

- Open access: articles freely available online

- High visibility within the field

- Retaining the copyright to your article 